\title{
Creencias sobre las drogas: diferencias según su consumo en adolescentes
}

\author{
Beliefs about drugs: Differences according \\ to own consumption by adolescents
}

\author{
Bárbara de los Ángeles Pérez Pedraza, Carla Mendoza Trejo, \\ Diana Isabel López Rodríguez y Verónica Alexandra Molina Coloma
}

\author{
Universidad Autónoma de Coahuila ${ }^{1}$
}

Autor para correspondencia: Bárbara Pérez Pedraza,barbara_perez@uadec.edu.mx.

\begin{abstract}
RESUMEN
La adolescencia es una etapa vulnerable en la que se inicia en el consumo de drogas. El objetivo del presente estudio comparativo fue contrastar las creencias y actitudes que tienen los adolescentes sobre las drogas, cuyo consumo es nulo o moderado; así como aquellos que realizan un consumo abusivo de tabaco, alcohol y drogas ilegales. Mediante un muestreo por conveniencia, se seleccionó a 50 participantes que reportaron un abuso de drogas, y otra de 51 participantes que afirmaron tener un consumo nulo o moderado, a los que se les aplicó el Cuestionario sobre Percepción de Riesgo de los Adolescentes hacia el Consumo de Drogas Legales e Ilegales. Los resultados indican que quienes se exceden en las drogas puntuaron más alto en creencias tales como que consumir bebidas alcohólicas ayuda a pasarla bien y provoca problemas escolares y laborales, comparados con los de los adolescentes con un consumo nulo o moderado. Se destaca la importancia de llevar a cabo intervenciones para la desnaturalización del uso de las drogas, de manera que los adolescentes comprendan cuándo su uso se convierte en abuso.
\end{abstract}

Palabras clave: Abuso de drogas; Actitudes; Creencias; Uso de drogas; Adolescentes.

\begin{abstract}
Adolescence is a vulnerable stage in which drug use begins. The objective of this study was to compare beliefs and attitudes about drugs in adolescents with no or moderate consumption and those abusing tobacco, alcohol, or illegal drugs. A total of 50 participants scored with abusive drug use. A sample of 51 participants who scored with zero or moderate consumption was selected by convenience sampling. Participants responded to the Risk Perception Questionnaire of Adolescents towards the Use of Legal and Illegal Drugs. The results indicate that those who abuse drugs scored higher in beliefs such as that consuming alcoholic beverages helps have a good time but causes school and work problems, compared to the beliefs of adolescents with zero or moderate consumption. Results underline the importance of interventions against perceiving drug use as natural, so adolescents understand when its use becomes abuse.
\end{abstract}

Key words: Drugs abuse; Attitudes; Beliefs; Drug use; Adolescents.

\footnotetext{
${ }^{1}$ Facultad de Psicología, Blvd. V. Carranza s/n, Col. República Oriente, 25280 Saltillo, Coah., México, tel. (844)438-16-00, correos electrónicos: barbara_perez@uadec.edu.mx, carlasmdz96@gmail.com,diana_lopez@uadec.edu.mx y molina@uadec.edu.mx.
} 
Recibido: $19 / 06 / 2020$

Aceptado: 07/10/2020

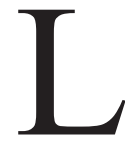

a última Encuesta Nacional de Consumo de Drogas, Alcohol y Tabaco (ENCODAT) (Comisión Nacional contra las Adicciones [CONADIC], 2017) muestra que de 2011 a 2017 hubo un aumento en el consumo excesivo o abuso de estas drogas, mientras que el consumo esporádico o controlado tanto de drogas legales como ilegales se mantuvo estable entre los adolescentes de 12 a 17 años, lo que hace de esta población un grupo vulnerable para el desarrollo de adicciones y otros problemas de salud física y mental.

A este respecto, se sabe que los adolescentes presentan una grave problemática, pues según los resultados de Villatoro et al. (2016), 30.4\% de adolescentes han consumido tabaco alguna vez en el paso de la secundara a la preparatoria, siendo la edad promedio de inicio de esta sustancia los 13.4 años. De ellos, $53.2 \%$ ha bebido alcohol, y $14.5 \%$ lo ha hecho en exceso. El consumo de drogas alguna vez fue de $17.2 \%$, siendo las drogas de preferencia la mariguana (10.6\%), los inhalables $(5.8 \%)$ y la cocaína $(3.3 \%)$.

Hoy en día se sabe que las adicciones son de origen multifactorial y que en el uso y abuso de drogas intervienen diversos elementos físicos, sociales, culturales e intrapersonales (Loor, Hidalgo, Macías, García y Srich, 2018); sin embargo, algunas investigaciones recientes se han centrado en las creencias y las actitudes que tienen los adolescentes sobre las drogas ya que han demostrado actuar como factores de riesgo para su mayor consumo (DiBello, Miller y Carey, 2019; Fox et al. 2017; Martínez, Vilar, Lozano y Verdejo, 2018).

De acuerdo con la teoría de la acción razonada, las actitudes y las creencias están íntimamente relacionadas, de manera que estas últimas se conforman en función a la asociación entre una conducta determinada y los posibles resultados de ejecutarla, los que pueden ser evaluados como buenos o malos. Así, las creencias dan origen a una actitud, es decir, la intención de llevar a cabo una conducta, la que puede ser positiva, negativa o neutral (Ajzen y Fishbein, 1980; $c f$. también Rodríguez, 2007).
Las creencias dependen del entorno; por ejemplo, en el caso del consumo de drogas, se observa que en la etapa de la adolescencia los jóvenes tienden a imitar a sus pares con la finalidad de crear un nuevo vínculo y así lograr pertenecer a un grupo determinado; en ocasiones, para ser aceptados, toman los valores y reglas establecidas dentro de estos grupos, que frecuentemente transmiten información errónea sobre el consumo de drogas.

Se han efectuado diferentes estudios para explicar lo que ocurre con las personas que las utilizan repetidamente con el propósito de comprender las creencias acerca de su consumo. Así, en una investigación de Martínez et al. (2018) hecha para evaluar las creencias nucleares sobre el consumo de drogas y el craving ${ }^{2}$, se encontró que las principales ideas que aparecen se agrupan así: lo que la persona cree que no podrá hacer en ausencia del efecto de la sustancia; la falta de renuncia al consumo, que gira en torno a la intención de volver a consumir; las condiciones que deberían darse para tomar la decisión de volver a consumir, y el propio consumo, que les permite sentirse bien consigo mismos y desarrollar sus potencialidades.

En su estudio con adolescentes, Fox et al. (2017) hallaron que algunas de las creencias que se tienen respecto al abuso de las drogas es que muchas de estas vienen adulteradas, que es difícil encontrar una dosis apropiada, y que las personas que abusan de estas no deberían tomar decisiones, entre otras creencias relacionadas al riesgo de neurotoxicidad. No obstante, los adolescentes que abusan de las drogas creen que les sirven para divertirse y "pasarla bien", para escapar o librarse de experiencias traumáticas, y para despertar o darse un trago de energía para calmar el dolor emocional.

Algunas personas creen que el consumo de sustancias ilegales les ayuda a mejorar su desempeño sexual y a interactuar más abiertamente con sus pares (Evers, Geraets, Van Liere, Hoebe y Dukers-

\footnotetext{
${ }^{2}$ Chesa, Elías, Fernández, Izquierdo y Sitjas (2004) señalan que “La Organización Mundial de la Salud, en 1955, englobó dentro del paraguas del craving las siguientes conductas: recaída, establecimiento de un uso excesivo de droga, pérdida de control y consumo diario excesivo. Así, propuso que el deseo o craving explicaba el consumo que aparecía de la necesidad psicológica y física, y de la necesidad de eliminar la abstinencia”.
} 
Muijrers, 2020; Fox et al., 2017). En efecto, numerosos adolescentes y adultos consideran que el uso de sustancias psicoactivas ayuda a potenciar la experiencia sexual y a desinhibir la conducta, por lo que consideran que les ayuda a socializar en situaciones en las que generalmente no podrían.

Hay otro tipo de creencias que subyacen al consumo de drogas, como lo son las religiosas y las educativas; de hecho, se ha encontrado que las creencias religiosas suelen estar relacionadas con el tipo de drogas que se consumen, siendo el alcohol la droga que aceptan más las personas con creencias cristianas (Thorens et al., 2016), así como que, aun cuando se considere que se ha recibido un adecuado conocimiento sobre las drogas y sus consecuencias, el consumo de alcohol, tabaco y marihuana sigue ocurriendo (De Souza, Cardoso, Marcolan, Vieira y Santos, 2018).

En México, se ha encontrado que algunas de las creencias respecto al consumo de las drogas están relacionadas con la cultura del narcotráfico, pues en el discurso de adolescentes y jóvenes se halla que la conceptualización positiva del narcotráfico como medio y estilo de vida propicia una concepción naturalizada del consumo de las drogas, las que se utilizan para encajar en la sociedad y tener una mejor posición social al venderlas y consumirlas (Barrera y Hernández, 2019).

Las creencias y actitudes respecto a las drogas están sumamente influidas por la percepción de ciertos elementos protectores y de riesgo; por ejemplo, se ha detectado como un factor de protección ante las drogas la satisfacción de las necesidades de disfrute asociadas, como la risa, el canto y el baile, que se producen en la interacción social, o la ejecución de actividades recreativas y físicas. Por otro lado, son factores de riesgo no cumplir con las necesidades de afecto, destacando el factor interrelacional y social como una influencia en las creencias sobre las drogas, así como las relaciones familiares rígidas o desligadas, los vínculos con amistades que consumen drogas y un pobre nivel educativo (Hernández, Fernández, Zapata, Crespo y González, 2019; Ortiz y Ortiz, 2018).

Las creencias que tienen los hombres y mujeres respecto a los factores de riesgo en el consumo de drogas son similares. Herruzo, Lucena,
Ruiz, Raya y Pino (2016), por ejemplo, detectaron que $85.5 \%$ de los hombres y $87.9 \%$ de las mujeres percibe que el mayor peligro del consumo es en la salud; los hombres, en concreto, consideran que se tienen más conflictos legales y más accidentes de tráfico $(82.9 \%)$, y las mujeres que surgen más complicaciones en las relaciones interpersonales (84.4\%).

Otros estudios recientes se refieren al consumo de drogas como un factor que les abre la oportunidad a los adolescentes para obtener un mayor respeto en un contexto hostil marcado por la desigualdad, sobre todo entre los sectores vulnerables, aunque se sospecha que lo mismo ocurre en estratos sociales altos, en los que se encubren los problemas relacionados con las drogas (Ospina, 2019). De modo que se destaca el contexto, las elecciones y sobre todo el desarrollo social de los adolescentes en el ambiente familiar y emocional.

En donde esta etapa del desarrollo propicia resultados más desalentadores sucede en la edad adulta por las experiencias más frecuentes de consumo y abuso de drogas licitas e ilícitas, por los cuales se desarrollan las adicciones como un estilo de vida, e incluso se perciben como normales, sin mostrar interés por la salud.

Como se observa, las creencias que rodean el consumo de drogas determinan con mucho desde los aspectos negativos que conllevan, hasta los beneficios sociales, sexuales y emocionales que se perciben como resultado de su uso, los factores de riesgo y protectores, así como las creencias relacionadas con los entornos sociales y culturales, como los religiosos, los educativos y el narcotráfico mismo.

Dadas las características de las drogas, el CONADIC (2017) ha hecho una diferenciación para identificar si se está usando o abusando de una de ellas según la cantidad, la frecuencia y las consecuencias del consumo, midiéndolas con indicadores como la ingesta, sus patrones de consumo y los problemas asociados al mismo, como enfermedades físicas o mentales, accidentes, conductas antisociales y dificultades para llevar a cabo las actividades de la vida diaria.

La Organización Mundial de la Salud (OMS, 1994) ofrece la definición para el consumo o uso 
experimental, que se refiere a las primeras veces que se experimenta con las drogas y que implica un consumo extremadamente infrecuente o inconstante de drogas ilegales, o el consumo controlado o social de alcohol o tabaco.

Se caracteriza el abuso de sustancias por el consumo continuo de la sustancia psicoactiva, a pesar de que la persona sabe que tiene dificultades en algún aspecto de su vida derivadas de dicho consumo, además de que le resulta físicamente arriesgado. Otro criterio implica que estos síntomas persisten por lo menos un mes, o bien que han aparecido repentinamente por un tiempo prolongado, sin que la persona cuente con un diagnóstico de dependencia (CONADIC, 2017).

En el caso de las drogas ilegales, como la cocaína, el crack, las anfetaminas y metanfetaminas, éxtasis, derivados del opio, tranquilizantes, inhalables, marihuana, peyote, hongos, LSD, entre otras, es común considerar que cualquier uso, independientemente de la cantidad consumida, es un abuso de la sustancia. Sin embargo, respecto a las drogas legales, como el alcohol y el tabaco, se establecen otros estándares.

Esta diferenciación conceptual entre el consumo moderado y el abuso de sustancias desempeña un papel fundamental para la implementación de programas de primero, segundo y tercer niveles, en donde desde el abordaje de la psicología de la salud se trabaja cognitivamente con las creencias y actitudes ante las drogas, así como con otras variables relacionales que favorecen las conductas de consumo no saludables.

Es con base en lo anteriormente dicho que el objetivo de este trabajo fue identificar el consumo de tabaco, alcohol y drogas ilegales, así como comparar las creencias y actitudes sobre las drogas en adolescentes que tienen un consumo nulo o moderado y quienes tienen un consumo abusivo de las drogas.

\section{MÉTODO}

Se llevó a cabo un estudio transversal comparativo con el objetivo de contrastar las creencias y actitudes de los adolescentes respecto al consumo de drogas en función de su consumo de drogas legales o ilegales, ya sea mediante el uso moderado o excesivo.

\section{Participantes}

Mediante un muestreo no probabilístico por conveniencia, se tomó una muestra inicial de 260 estudiantes de un bachillerato de la ciudad de Saltillo (México), a los que se les solicitó una carta de consentimiento informado firmada por sus padres o tutores para la utilización de sus respuestas para fines de investigación.

De dicha muestra inicial, se seleccionaron aquellos que puntuaron con un consumo desmedido de tabaco, alcohol u otras drogas, de acuerdo con los criterios establecidos por el CONADIC (2017):

Alcohol: Presencia de consumo de moderado a alto de alcohol con problemas de moderados a severos.

Tabaco: Fumador diario /o consumo de moderado a alto.

Drogas ilegales: Cualquier consumo que no sea de primera vez.

Así, se recabó un total de 50 adolescentes con consumo abusivo de alguna de estas sustancias o de dos o más de ellas, de los que $57.8 \%$ fueron mujeres, con una edad media de 15.73 años.

Con el propósito de igualar la muestra de adolescentes con consumo abusivo, se seleccionó una segunda muestra de adolescentes con consumo moderado o nulo de drogas, tomando en cuenta los criterios por la OMS (1994):

Alcohol y tabaco: Consumo moderado y sin intoxicación. ilegal.

Drogas ilegales: Consumo único de la droga

Se seleccionó solamente a aquellos adolescentes que respondieron a 95\% del cuestionario, excluyendo a quienes omitieron más de $5 \%$ de las respuestas, quedando un total de 51 participantes con consumo moderado de drogas, de los que $53.8 \%$ fueron hombres, con una edad media de 15.44 años. 


\section{Instrumentos}

Cuestionario sobre Percepción de Riesgo de los adolescentes hacia el consumo de drogas legales e ilegales (Uribe, Verdugo y Zacarías (2011).

Este cuestionario, basado en el Cuestionario de Percepción de Riesgo y Consumo de Drogas de Salazar, Varela, Tovar y Cáceres (2006), fue validado para población mexicana y mide el consumo de drogas legales e ilegales, así como los factores de riesgo y de protección a las adicciones en los adolescentes. Está conformado por seis apartados, de los que se retomaron tres: el primero recoge los datos sociodemográficos; el segundo pregunta por la frecuencia de consumo de tabaco, alcohol y drogas ilegales, así como la edad de inicio en el consumo de estos; cuenta con 18 ítems que se valoran en una escala tipo Likert de cinco opciones de respuesta que van de "nada" a "bastante", y el tercero pregunta por las creencias acerca del consumo de drogas ilegales, conformado por 30 reactivos que se responden con una escala Likert de cinco opciones de respuesta que van de "totalmente desacuerdo" a "totalmente de acuerdo".

\section{Procedimiento}

Se solicitó el permiso en una institución de bachillerato para realizar el estudio. Una vez obtenido, se remitieron las cartas de consentimiento informado y el aviso de privacidad a los padres o tutores de los menores, en las que se les garantizó la confidencialidad de los datos y su uso para fines de investigación.

El día de la aplicación se recogieron las cartas con los formatos firmados por los padres o tutores, proporcionándoles el cuestionario únicamente a los estudiantes que los entregaron. La aplicación se llevó a cabo grupalmente y de forma guiada por personas capacitadas.

\section{Análisis de datos}

Se utilizó el programa estadístico SPSS-24 para el procesamiento de datos, llevándose a cabo análisis descriptivos para la caracterización de la muestra, así como la prueba $t$ de Student para muestras independientes y la $d$ de Cohen para medir el tamaño

Instituto de Investigaciones Psicológicas - Universidad Veracruzana del efecto. Asimismo, se efectuó un examen de análisis de varianza (ANOVA) con contrastes post hoc con el estadístico C de Dunnet en función de la no homogeneidad de varianzas del valor $p$ de Levene, todo esto asumiendo una distribución paramétrica con base en el teorema central del límite.

\section{RESULTADOS}

Se puede observar en la Tabla 1 que casi ninguno de los adolescentes había consumido droga alguna, y que el resto solamente alcohol. Por otro lado más de una tercera parte de quienes tuvieron consumo abusivo, en este caso alcohol, seguido por una proporción menor de consumo de una combinación de dos o más drogas legales o ilegales.

Tabla 1. Consumo moderado y abusivo de drogas.

\begin{tabular}{|l|r|r|r|}
\cline { 2 - 3 } \multicolumn{1}{c|}{} & \multicolumn{2}{c|}{ Consumo } & \multirow{2}{*}{ Total } \\
\cline { 2 - 3 } \multicolumn{1}{c|}{} & $\begin{array}{c}\text { Moderado } \\
\boldsymbol{f}(\mathbf{\%})\end{array}$ & $\begin{array}{c}\text { Abuso } \\
\boldsymbol{f}(\mathbf{\%})\end{array}$ & \multicolumn{1}{c|}{$(\mathbf{\%})$} \\
\hline Ninguna & $50(98.00)$ & $0(0)$ & $50(49.5)$ \\
\hline Tabaco & $0(0)$ & $5(10.00)$ & $5(5.00)$ \\
\hline Alcohol & $1(2.00)$ & $19(38.00)$ & $20(19.80)$ \\
\hline Ilegales & $0(0)$ & $8(16.00)$ & $8(7.90)$ \\
\hline Varias & $0(0)$ & $18(36.00)$ & $18(17.80)$ \\
\hline
\end{tabular}

En la Tabla 2 se observan las diferencias significativas entre los adolescentes con consumo moderado de drogas y aquellos con un consumo abusivo, encontrando que estos últimos puntuaron más alto en todas las creencias y actitudes respecto a las drogas.

En particular, el tamaño del efecto fue mayor en los adolescentes que abusaban de las drogas respecto a creer que consumir bebidas alcohólicas ayuda a "pasarla bien", que provoca problemas escolares y laborales, genera delitos y violencia y problemas en los estudios, y a tener una actitud negativa hacia las drogas porque crean problemas personales, familiares, laborales y escolares.

De igual modo, mostraron un tamaño del efecto mediocre en las diferencias de creencias y actitudes hacia las drogas los adolescentes con un consumo moderado y aquellos con un consumo en exceso, siendo estos últimos quienes puntua- 
Tabla 2. Diferencia de creencias y actitudes respecto a las drogas en función del nivel de consumo.

\begin{tabular}{|l|c|c|c|c|}
\hline \multicolumn{1}{|c|}{ Creencias y actitudes } & $\begin{array}{c}\text { Consumo } \\
\text { moderado } \\
\text { M (D.E.) }\end{array}$ & $\begin{array}{c}\text { Consumo } \\
\text { abusivo } \\
\text { M (D.E.) }\end{array}$ & $p$ & d \\
\hline $\begin{array}{l}\text { El consumo de bebidas alcohólicas genera problemas } \\
\text { en los estudios. }\end{array}$ & $2.35(1.34)$ & $3.22(1.22)$ & 0.001 & -.68 \\
\hline Por consumir bebidas alcohólicas hay inasistencia en clases. & $2.59(1.25)$ & $3.37(1.39)$ & 0.004 & -.59 \\
\hline $\begin{array}{l}\text { Consumir bebidas alcohólicas genera conflictos } \\
\text { con los padres. }\end{array}$ & $2.02(1.06)$ & $2.7(1.34)$ & 0.006 & -.56 \\
\hline $\begin{array}{l}\text { Las discusiones de pareja son a causa de consumir bebidas } \\
\text { alcohólicas. }\end{array}$ & $2.65(1.21)$ & $3.31(1.17)$ & 0.007 & -.55 \\
\hline $\begin{array}{l}\text { La gente que consume bebidas alcohólicas pierde el control } \\
\text { y es desagradable. }\end{array}$ & $2.22(1.15)$ & $2.87(1.45)$ & 0.014 & -.49 \\
\hline El consumo de bebidas alcohólicas provoca delitos y violencia. & $1.98(1.01)$ & $2.78(1.27)$ & 0.001 & -.70 \\
\hline $\begin{array}{l}\text { El consumo de bebidas alcohólicas provoca } \\
\text { efectos negativos en la vida familiar. }\end{array}$ & $1.96(1.08)$ & $2.41(1.06)$ & 0.039 & -.42 \\
\hline Consumir bebidas alcohólicas ayuda a pasarla bien. & $2.5(1.28)$ & $3.56(1.13)$ & 0.000 & -.88 \\
\hline $\begin{array}{l}\text { Se consume bebidas alcohólicas para mejorar } \\
\text { las relaciones interpersonales. }\end{array}$ & $2.12(1.14)$ & $2.60(1.24)$ & 0.047 & -.40 \\
\hline $\begin{array}{l}\text { Consumir bebidas alcohólicas provoca problemas escolares } \\
\text { y laborales. }\end{array}$ & $2.25(1.05)$ & $3.06(1.18)$ & 0.000 & -.73 \\
\hline Tomar bebidas alcohólicas es malo para la salud. & $1.80(1.06)$ & $2.42(1.20)$ & 0.007 & -.55 \\
\hline $\begin{array}{l}\text { El consumo de drogas legales e ilegales } \\
\text { crea adicción. }\end{array}$ & $1.55(.94)$ & $2.00(1.06)$ & 0.027 & -.45 \\
\hline $\begin{array}{l}\text { Nunca he consumido drogas porque crea problemas } \\
\text { personales, familiares, laborales y escolares. }\end{array}$ & $1.80(1.26)$ & $2.64(1.40)$ & 0.002 & -.63 \\
\hline
\end{tabular}

ron más alto en las creencias de que el consumo de bebidas alcohólicas propicia la inasistencia a las clases, que genera conflictos con los padres y la pareja, que provoca efectos negativos en la vida familiar, que es malo para la salud, que la gente que consume bebidas alcohólicas pierde el control y es desagradable, que el consumo de drogas legales e ilegales crea adicción, y que se consumen bebidas alcohólicas para mejorar las relaciones interpersonales.

En la Tabla 3 se puede observar que hubo diferencias significativas intergrupos en tres variables, según las cuales los adolescentes que abusaban del consumo de varias drogas puntuaron más alto en la creencia de que se consumen bebidas alcohólicas por diversión, comparados con quienes abusan únicamente del tabaco.

Tabla 3. Diferencias de creencias y actitudes en función de la droga de la que se abusa.

\begin{tabular}{|c|c|c|c|c|c|}
\hline Creencia & Droga-Droga & M & $\begin{array}{l}\text { Prueba } d \\
\text { de Levene }\end{array}$ & ANOVA & $p$ \\
\hline $\begin{array}{l}\text { Consumir bebidas alcohólicas } \\
\text { se hace por diversión }\end{array}$ & $\begin{array}{l}\text { Tabaco* } \\
\text { Alcohol } \\
\text { Ilegales } \\
\text { Varias* }\end{array}$ & $\begin{array}{l}2.20 \\
3.26 \\
3.38 \\
3.94\end{array}$ & .762 & .033 & .028 \\
\hline $\begin{array}{l}\text { Consumir bebidas alcohólicas } \\
\text { provoca problemas escolares } \\
\text { y laborales }\end{array}$ & $\begin{array}{l}\text { Tabaco* } \\
\text { Alcohol } \\
\text { Ilegales } \\
\text { Varias* }\end{array}$ & $\begin{array}{l}1.80 \\
3.05 \\
2.50 \\
3.67 \\
\end{array}$ & .455 & .004 & .007 \\
\hline $\begin{array}{l}\text { Se consumen drogas legales } \\
\text { para tener más amigos y ser } \\
\text { más sociable }\end{array}$ & $\begin{array}{l}\text { Tabaco* } \\
\text { Alcohol } \\
\text { Ilegales } \\
\text { Varias* }\end{array}$ & $\begin{array}{l}4.60 \\
2.94 \\
3.25 \\
2.39\end{array}$ & .334 & .010 & .006 \\
\hline
\end{tabular}

Instituto de Investigaciones Psicológicas - Universidad Veracruzana ISSN impreso: 1405-1109
Psicología y Salud, Vol. 32, Núm. 1: 115-123, enero-junio de 2022 https://doi.org/10.25009/pys.v32i1.2718 
Lo mismo ocurrió con la creencia de que consumir bebidas provoca problemas escolares y laborales, pues los adolescentes que abusaban del consumo de varias drogas puntuaron más alto en el grado de acuerdo con esta variable, comparados con quienes solo abusaban del tabaco. Finalmente, los adolescentes cuyo consumo de tabaco era excesivo puntuaron más alto en la creencia de que se consumen drogas legales para tener más amigos y ser sociable, comparados con aquellos que abusaban de varias drogas.

\section{DISCUSIÓN}

El uso y el abuso del consumo de drogas legales e ilegales por los adolescentes constituye hoy en día un problema de salud nacional (CONADIC, 2017). Por consiguiente, se resalta que en la muestra de adolescentes de esta investigación, la mayoría de los que afirmaron tener un consumo moderado es porque no habían consumido ningún tipo de droga; sin embargo, el resto se excedía principalmente en el alcohol, o bien presentaba una comorbilidad en el abuso de tabaco, alcohol y drogas ilegales. Esto concuerda con los resultados de otros estudios hechos con adolescentes que revelan que entre más jóvenes inician en el consumo de alguna droga, más propensos se hacen a la comorbilidad en el abuso de estas, por ejemplo en el consumo de alcohol y marihuana y de tabaco con alcohol (Roche et al., 2019).

Al dividir a los adolescentes con un consumo nulo o moderado y a aquellos con un consumo abusivo, se pudo efectuar el análisis de sus creencias y actitudes, encontrando que los adolescentes con consumo abusivo de drogas creen en mayor medida que el alcohol tiene efectos negativos en aspectos sociales, relacionales, familiares, escolares, cognitivos y sobre la salud; aun así, consideran que el alcohol les ayuda a "pasarla bien", lo que contrasta con el modelo propuesto por DiBello et al. (2019), según el cual el consumo abusivo de alcohol se explica en función de creencias que lo normalizan durante la adolescencia, así como por actitudes positivas respecto a embriagarse en el futuro. No obstante, estos resultados concuerdan con los de Fox et al. (2017), quienes hallaron que

Instituto de Investigaciones Psicológicas - Universidad Veracruzana a pesar de que quienes abusan de las drogas son quienes tienen un mayor conocimiento y datos específicos de la composición de las sustancias que consumen y sus efectos adversos, son asimismo quienes tienen una perspectiva positiva de estas, pues creen que así pueden pasar un buen rato y mejorar sus relaciones interpersonales, lo que es un foco de atención porque encuentran más ganancias secundarias que desventajas, ya que, tal como se observó en el presente trabajo, la lista de las consecuencias adversas del consumo de drogas fue más extensa que la de sus consecuencias positivas.

Por tanto, es posible afirmar que los participantes en el estudio consumían drogas aun sabiendo sus efectos nocivos, lo que puede deberse a que no reconocían que su consumo era abusivo. Ya Molina, González, Montero y Gómez (2016) afirman que una de las principales complicaciones para la coordinación social y sanitaria en el terreno de las adicciones tiene que ver con la falta de reconocimiento y de identificación de los usuarios de drogas como drogodependientes, y de que quienes aún no lo son, tienen sin embargo un consumo abusivo y un mayor riesgo de llegar a serlo.

Lo anterior se comprueba en cuanto que el grupo de adolescentes que puntuó con un consumo abusivo de drogas refirió en mayor medida que no consumían drogas, lo que contradice sus respuestas respecto al número de veces que consumían tabaco, alcohol o drogas ilegales, y puede estar relacionado con la normalización de la cultura del narco y el consumo de drogas, pues se pierde la percepción de lo que es una droga y se normaliza su uso; por ejemplo, de la mariguana por ser natural, o del alcohol y tabaco por ser legales, así como por el uso de estos en entornos de esparcimiento y socialización (Barrera y Hernández, 2019). De manera que, una vez más, este grupo de adolescentes está en un alto riesgo de desarrollar adicciones al no reconocer su consumo abusivo.

Finalmente, se diferencian las creencias respecto a las drogas según el tipo de sustancia de la que se abusa, y así los adolescentes que abusaban de varias drogas tenían la creencia de que el alcohol les ayudaba a divertirse y a la vez les provocaba problemas escolares y laborales, mientras que los que abusaban del tabaco creían que consumir 
drogas legales les ayudaba a ser más sociables. Esto representa riesgo, pues la diferencia conceptual de las ventajas que encuentran ambos grupos en las drogas es muy tenue en virtud de que en ambos casos se creía que les ayudaban a mejorar su situación actual, como lo refiere Ospina (2019), pues concedían un mayor status a los adolescentes en su grupo de referencia.

Hay entre los adolescentes una cierta incongruencia en sus creencias cuando identifican el alcohol como una fuente de problemas escolares y laborales, pero ellos mismos lo consumen, al igual que otras drogas ilegales. Ya Martínez et al. (2018) refieren que las creencias nucleares de las personas que abusan de las drogas se centran en la experiencia placentera que les genera la sustancia, sin que establezcan una conexión con las otras áreas de su vida, por lo que parece haber una disociación entre su consumo y las consecuencias personales del mismo.

Es por ello que los adolescentes que pertenecían al grupo con consumo moderado no consumían ningún tipo de droga, mientras que los que abusaban de ellas lo hacían principalmente respecto al alcohol o a la combinación de drogas legales e ilegales. Este segundo grupo creía en los efectos negativos del uso de drogas en la salud y la familia, pero también en los efectos positivos sobre su estado de ánimo y en su interacción con sus pares; en efecto, quienes consumían sobre todo tabaco creían divertirse más y tener más amigos.
Es por lo anterior que se subraya la importancia de identificar las creencias y actitudes hacia el uso de drogas, pues los adolescentes, estando en una etapa tan vulnerable, están particularmente influidos por sus contextos inmediatos y su cultura, que normaliza tanto el consumo de drogas que les es difícil distinguir en qué momento se están excediendo, e incluso se les dificulta identificar qué sustancias son drogas y cuáles no. Asimismo, se acentúan en ellos las creencias acerca de la satisfacción inmediata, por encima de las creencias sobre los problemas relacionados con las drogas, por lo que constituyen un sector particularmente propenso a las adicciones y a otros problemas para su salud física y mental.

Finalmente, este estudio refuerza una línea de investigación respecto a la influencia de los pares y otros agentes sociales, como la publicidad, la sociedad y la misma familia, en el desarrollo de esas creencias y el consumo de las sustancias, pues como señalan García et al. (2008), en tales interacciones se originan gran parte de las creencias respecto al consumo de drogas. Resulta también necesario profundizar en las inconsistencias o contrastes encontrados en este estudio, sobre todo con los adolescentes que presentan un consumo abusivo de sustancias, de manera que se pueda comprender el proceso cognitivo que por un lado, les lleva a creer que las drogas afectan en varios aspectos de la vida, pero por otro lado siguen consumiéndolas en exceso.

Citación: Pérez P., B.Á., Mendoza T., C., López R., D.I. y Molina C., V.A. (2022). Creencias sobre las drogas: Diferencias según el consumo de estas en adolescentes. Psicología y Salud, 32(1), 115-123. https:// Doi.org/10.25009/pys.v32i1.2718.

\section{REFERENCIAS}

Ajzen, I. y Fishbein, M. (1977). Attitude-behavior relations: A theoretical analysis and review of empirical research. Psychological Bulletin, 84, 888-918.

Barrera, A. y Hernández D. (2019). Fascinación por el poder: consumo y apropiación de la narco cultura por jóvenes en contextos de narcotráfico. Intersticios Sociales, 17, 259-285.

Chesa V., D., Elías A., M., Fernández V., E., Izquierdo M., E. y Sitjas C., M. (2004). El craving, un componente esencial en la abstinencia. Revista de la Asociación Española de Neuropsiquiatría, 89. Recuperado de http://scielo.isciii.es/scielo.php?script $=$ sci_arttext\&pid=S0211-57352004000100007.

Comisión Nacional contra las Adicciones (2017). Prevención de las adicciones y promoción de conductas saludables para una nueva vida; guía para el promotor de "nueva vida”. México: CONADIC, Secretaría de Salud.

De Souza, L., Cardoso, T., Marcolan, F., Vieira, S. y Santos, M. (2018). El fenómeno de las drogas en la perspectiva de los estudiantes de enfermería: perfil del consumo, actitudes y creencias. Escola Anna Nery, 22(1), 1-8. Doi: 10.1590/2177-9465EAN-2017-0205. 
DiBello, A., Miller, M. y Carey, K. (2019). Positive heavy drinking attitude mediates the association between college alcohol beliefs and alcohol-related outcomes. Addictive Behaviors, 88, 29-35. Doi: 10.1016/j.addbeh.2018.08.005.

Evers, Y., Geraets, J., Van Liere, G., Hoebe, C. y Dukers-Muijrers, N. (2020). Attitude and beliefs about the social environment associated with chemsex among MSM visiting STI clinics in the Netherlands: An observational study. PlosOne, 15(7), 1-12. Doi: 10.1371/journal.pone.0235467.

Fox, J., Smith, A., Yale, A., Chow, C., Alaswad, E., Cushing, T. y Monte, A. (2017). Drugs of abuse and novel psychoactive substances at outdoor music festivals in Colorado. Substance Use \& Misuse, O(0), 1-9. Doi: 10.1080/10826084.2017.1400067.

García A., R., Rodríguez K., S. y Córdova, A. (2008). Factores motivacionales protectores de la depresión y el consumo de drogas. Salud Mental, 31(6), 453-459.

Hernández, D., Fernández, C., Zapata, J., Crespo, M. y González, N. (2019). Caracterización cognitiva y conductual de una muestra de adolescentes consumidores de inhalables de la ciudad de México. Health and Addictions, 19(2), 19-28.

Herruzo, C., Lucena, V., Ruiz O., R., Raya, A. y Pino, M. (2016). Diferencias en función del sexo en la percepción del riesgo asociado al consumo de drogas en jóvenes. Acción Psicológica, 13(1), 79-90. Doi: 10.5944/ap.13.1.17422.

Loor, W., Hidalgo, H., Macías, J., García, E. y Srich, A. (2018). Causas de las adicciones en adolescentes y jóvenes en Ecuador. Archivo Médico Camagüey, 2(22), 130-138.

Martínez G., J., Vilar, R., Lozano R., O. y Verdejo G., A. (2018). Cuestionario de creencias nucleares relacionadas con el consumo de drogas y el craving, para la valoración del riesgo de recaída. Adicciones, 30(3), 170-178.

Molina, A., González, J., Montero, J. y Gómez S., J. (2016). La complejidad de la coordinación social y sanitaria en las adicciones y el papel de la enfermera. Enfermería Clínica, 1, 68-75. Doi: 10.1016/j.enfcli.2015.09.009.

Organización Mundial de la Salud (1994). Glosario de términos de alcohol y drogas. Ginebra: OMS.

Ortiz, M. y Ortiz M., M. (2018). Factores de riesgo en adicciones y su relación con actividades deportivas y recreativas. Retos, 34, 71-79.

Ospina, A. (2019). Entre el orgullo y la vergüenza. El espectro emocional en las biografías de varones que se inyectan drogas en Hermosillo, Sonora. Cultura y Representaciones Sociales, 26(1), 337-372.

Roche, D., Bujarski, S., Green, R., Hartwell, E., Leventhal, A. y Ray, L. (2019). Alcohol, tobacco, and marijuana consumption is associated with increased odds of same-day substance co-and tri-use. Drug and Alcohol Dependence, 200, 40-49. Doi: 10.1016/j.drugalcdep.2019.02.035.

Rodríguez, L. (2007). La teoría de acción razonada: implicación de estudio de las actitudes. Investigación Educativa, 7, 66-77.

Salazar I., C., Varela M., T., Tovar J., R. y Cáceres D., E. (2006). Construcción y validación de un cuestionario de factores de riesgo y de protección para el consumo de drogas en jóvenes universitarios. Acta Colombiana de Psicología, 9(2), 19-30.

Thorens, G., Rothen, S., Gmel, G., Khazaal, Y., Achab, S., Calzada, G. y Zullino, D. (2016). Association between religious and drugs of choice in a Swiss male sample. Journal of Addictive Behaviors: Therapy \& Rehabilitation, 5(1), 1-12. Doi: 10.4172/2324-9005.1000153.

Uribe J., I., Verdugo J., C. y Zacarías, X. (2011). Relación entre percepción de riesgo y consumo de drogas en estudiantes de bachillerato. Psicología y Salud, 21(1), 47-55.

Villatoro, J., Medina-Mora, M., Campo, R., Fregoso, D., Bustos, M., Reséndiz, E., Mujica, R. Cañas, V. (2016). El consumo de drogas en estudiantes de México: tendencias y magnitud del problema. Salud Mental, 39(4), 193-203. Doi: 10.17711/ SM.0185-3325.2016.023. 